\title{
$x=-1-2=-2$ \\ Unmixing AVHRR Imagery to Assess Clearcuts and Forest Regrowth in Oregon
}

\author{
Christine A. Hlavka and Michael A. Spanner
}

\begin{abstract}
Advanced Very High Resolution Radiometer imagery provides frequent and low-cost coverage of the earth, but its coarse spatial resolution $(\tau 1.1 \mathrm{~km}$ by $1.1 \mathrm{~km})$ does not lend itself to standard techniques of automated categorization of land cover classes because the pixels are generally mixed; that is, the extent of the pixel includes several land use/cover classes. Unmixing procedures were developed to extract land use/cover class signatures from mixed pixels, using Landsat Thematic Mapper data as a source for the training set, and to estimate fractions of class coverage within pixels. Application of these unmixing procedures to mapping forest clearcuts and regrowth in Oregon indicated that unmixing is a promising approach for mapping major trends in land cover with AVHRR bands 1 and 2. Including thermal bands by unmixing AVHRR bands 1-4 did not lead to significant improvements in accuracy, but experiments with unmixing these four bands did indicate that use of weighted least squares techniques might lead to improvements in other applications of unmixing.
\end{abstract}

\section{INTRODUCTION}

$\mathbf{T}$ HE Advanced Very High Resolution Radiometer (AVHRR) series of sensors aboard the National Oceanic and Atmospheric Administration (NOAA) polarorbiting satellites was originally designed for meteorological applications, in particular, tracking of weather (especially cloud) patterns and estimation of sea-surface temperatures using the thermal bands $(3550-3930 \mathrm{~nm}, 10300-11300$ $\mathrm{nm}$, and $11500-12500 \mathrm{~nm}$ ). In recent years, however, AVHRR imagery has been used to monitor the land's surface, especially vegetation dynamics at regional to global scales [1], [2] by using the reflective bands $(580-680 \mathrm{~nm}$ in the visible red, $725-1100 \mathrm{~nm}$ in the near-infrared). This imagery lends itself to large-area monitoring applications because of its low cost, wide-area coverage (over a million square kilometers per scene), frequency of acquisition (twice per day), and useful bands for monitoring land processes.

The spatial resolution of AVHRR, about $1.1 \mathrm{~km}$, precludes monitoring land cover or mapping landscape features with the traditional techniques of image classification because patterns of land cover types are typically of a similar or smaller size. For example, a standard size for agricultural fields in the United States is 160 acres (or 65 ha, about 53\% of an AVHRR pixel). Clearcut patches in western forests generally range from several hectares to one square kilometer. Patterns like these are

Manuscript received February 25, 1993; revised January 10, 1995. This research was supported in part by NASA's Biospheric Research Program.

C. A. Hlavka is with NASA Ames Research Center, Moffett Field, CA 94035-1(H)( USA

M. A. Spanner is with Johnson Controls World Services, Inc.. NASA Ames Research Center, CA 94035-1000 USA.

IEEE L.og Number 9411241 readily observed and mapped with Landsat Thematic Mapper (TM) imagery, with a resolution of $30 \mathrm{~m}$, but TM data are expensive to acquire and process (due to high data volume), and coverage is relatively infrequent, due to the 16-day repeat cycle of the Landsat orbit and frequent cloud obscuration.

It has been proposed that regional studies of land surface processes involve a combination of low spatial resolution sensors, such as AVHRR, and high-resolution sensors, such as TM; several investigations have tested this approach [3], 14]. Generally, the idea is that AVHRR data provide complete coverage spatially and/or temporally, while TM data provide detailed information in sample areas for "calibrating" the AVHRR data. Unmixing techniques, which model pixels as mixtures of pure components, potentially are useful in such a scheme since AVHRR pixels are often mixed, while TM imagery can provide information for fitting the model.

The study of biogeochemical cycling in the coniferous forests of the western United States is an example of the type of regional study that can potentially benefit from the approach described above. Because of logging practices, many of these forests are a patchwork of clearcuts of varying ages and sizes. Disturbances such as clearcutting affect cycling of nutrients. For example, newly disturbed sites in U.S. forests have been shown to be susceptible to losses of nitrate, nitrogen gas, and nitrous oxide [5]-17]. Increased fluxes of carbon dioxide to the atmosphere following disturbance have been observed [8]. Once uptake of nutrients by vegetation has resumed, nutrient losses are substantially reduced.

There are three major stages of forest disturbance and regrowth which are potentially observable with remotely sensed imagery: newly disturbed or clearcut with mostly bare soil, substantial regrowth where successional vegetation (shrubs, brush, and young trees) dominate, and reestablishment of coniferous canopy cover greater than $75 \%$. The objective of the work reported here was to develop procedures for unmixing AVHRR using sample TM data. These techniques were applied to mapping the three classes described above to test their utility in detection, quantification, and mapping of forest disturbance.

\section{THE STUDY AREA}

The study area, shown in Figs. 1 and 2, was centered on the H. J. Andrews Experimental Forest, a 6000-ha watershed located on the western slopes of the Oregon Cascade range. The mixed-species coniferous forests at $\mathrm{H}$. J. Andrews are principally Douglas fir (Pseudotsuga menziesii), western hemlock (Tsuga heterophylla), and western red cedar (Thuja plicata) in the lower elevations $(400-1050 \mathrm{~m})$ and Pacific 


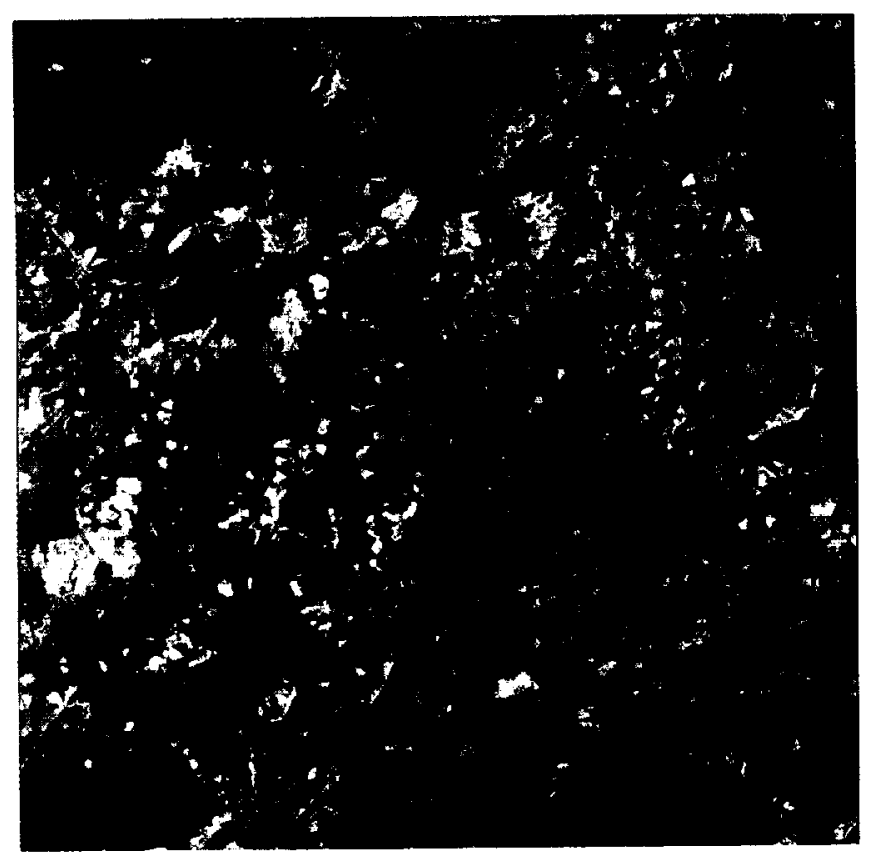

Fig. 1. False color composite $(\mathrm{RGB}=$ bands $1,2,4)$ of Landsat $\mathrm{TM}$ coverage of the study site, H. J. Andrews Experimental Forest and environs. The mature forest appears brown; clearcuts are blue; patches of successional vegetation appear red/pink.

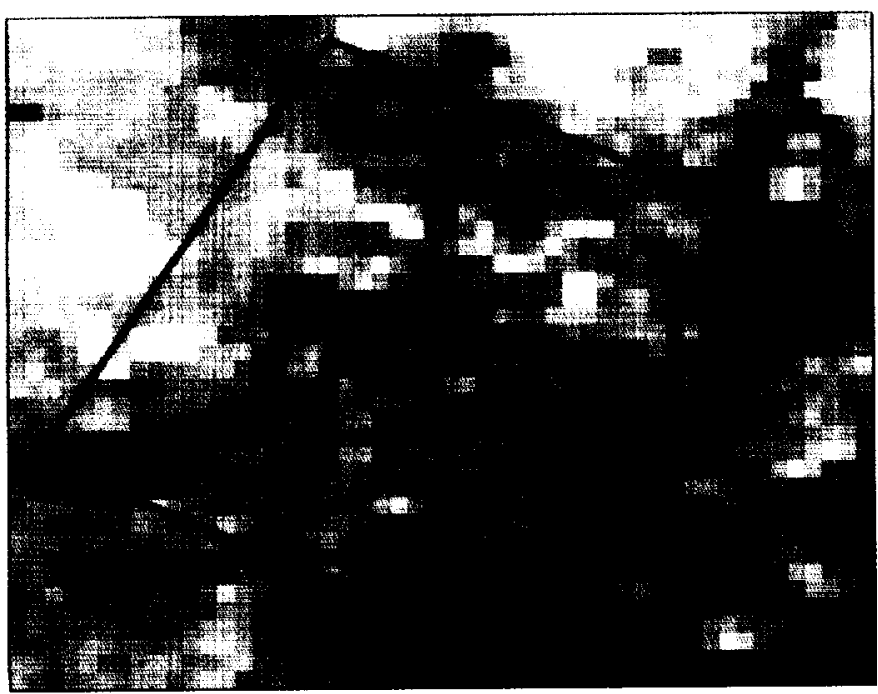

Fig. 2. False color composite ( $\mathrm{RGB}=$ bands $1,2,3$ ) of AVHRR coverage of the study site. Boundaries delineate the area corresponding to Landsat TM as pictured in Fig. 1.

silver fir (Abies amabalis) and western hemlock at higher elevations $[9]$.

Extensive logging began in 1950 and involved two methods: 1) salvage logging in which decadent trees were harvested from old-growth stands, and 2) clearcut logging where all trees were cut from sites ranging from 4 to 16 ha in area. Following logging, many of the clearcut sites were burned to remove slash and some were replanted with conifer seedlings. The clearcut sites were delineated by U.S. Forest Service researchers on a map of the $\mathrm{H}$. J. Andrews Experimental Forest. In addition, the years of logging and revegetation of clearcut sites were listed on H. J. Andrews stand history documents [10] which reference the map. We visited and photographed most of the sites in 1987; percent ground cover of vegetation regrowth were estimated visually [11].

\section{METHODS}

\section{A. The Mixture Model}

Several investigators [12]-[15] have analyzed remotely sensed imagery by estimating component proportions of mixed pixels using a least squares approach. For our application, the components are the three forest disturbance classes ( 1 -clearcut, 2 -forest of $75 \%$ crown closure, and 3 - succession). The model for the radiance $(L)$ of a pixel $(p)$ in band $(b)$ is

$$
\begin{aligned}
L(p, b)= & M 1(b) * F 1(p)+M 2(b) * F 2(p)+M 3(b) \\
& * F 3(p)+e(p, b)
\end{aligned}
$$

subject to the constraint

$$
F 1(p)+F 2(p)+F 3(p)=1
$$

where $M 1(b), M 2(b)$, and $M 3(b)$ are the mean $L$ values for classes 1, 2, and 3 in band $l$; and $F 1(p), F 2(p)$, and $F 3(p)$ are the fractions of the three classes. The error term $e(p, b)$ is a term representing the combined effect of local deviations of $L$ values of the components from their average values. In this study, the $L$ values were the digital numbers (DN values) of the imagery; since DN values are linear functions of radiance [16], (1) is still applicable.

Three assumptions determine the applicability of this model: 1) The land use/land cover classes each have a fairly distinct spectral signature. In the case of the clearcut, forest and succesion classes, this was presumed to be true because they are associated with three morphologically distinct associations of vegetation (soil/grasses, shrubs/small trees, mature coniferous trees) and because of their appearance in TM imagery (Fig. 1). 2) The scene is presumed to consist of a mosaic of patches of the three cover types, as a result of clearcuts in an area that was originally all forest. Fig. 1 shows that this presumption is nearly true, although there are some small areas of water and road. Factors 1) and 2) are the same as with applicability of supervised classification. 3) The resolution of the AVHRR sensors are all about the same, with a nominal resolution of about $1.1 \mathrm{~km}$.

The linearity of mixing is due to the process of image formation by the AVHRR instrument. When atmosperic conditions are fairly clear, radiance at the sensor is approximately the convolution of the radiance from the scene with the point-spread function (PSF) of the sensor. When the three assumptions above are true, then the result of the convolution is approximated by (1), with consistent values for $F 1(p)$. $F 2(p), F 3(p)$ for all bands (because of factor 3 ). The model will be useful, that is, the error term $e(p, b)$ will be small relative to the sum of the other terms, which will be the case if assumption 1 (separability of classes) holds. 


\section{B. Signature Development}

The study area contained no patches of pure undisturbed forest, clearcut, or successional stage that were several kilometers in extent, therefore it was not possible to estimate AVHRR signatures of these classes using the standard methods associated with image classification. The AVHRR signatures of the forest classes were instead derived through least squares fitting of the mixture model. The TM data were used to compute a training set of fractions on a portion of the study area. Multivariate regression was then applied to the training fractions and corresponding AVHRR radiance values to derive least squares estimates of $M 1(b), M 2(b)$, and $M: 3(b)$. The covariance of $e(p, b)$ was also estimated.

The data for the regression were developed from classified TM imagery. A TM scene acquired on July 10, 1984 was carefully examined to select a rectangular subimage including the H. J. Andrews Experimental Forest while avoiding areas with agricultural development and some large lava beds in the vicinity of the Forest. This subimage covered approximately 110000 ha. The subimage was stratified into the three forest disturbance cover classes by performing a supervised maximum-likelihood classification. The training sites were 30 of the clearcut sites in the H. J Andrews Experimental Forest, which were selected to be representative of the ranges of clearcut ages and locations within the Forest. The classification was quality checked by visual comparison with a color infrared aerial photograph acquired from a 1983 flight of NASA/Ames ER-2 aircraft at a scale of 1:65000.

The classified TM data were then processed to create three fraction images which were then registered to AVHRR imagery. A binary image for each cover class (with l's for pixels in the class, 0's elsewhere) was created by recoding the clustered image. Each binary image was convolved with a Gaussian filter (approximating the point-spread function, or "footprint," of AVHRR) to compute weighted averages of the pixels (1's and 0 's) over an area the size of an AVHRR pixel $|17|$. Since the study area was located within the center half of the range of the AVHRR scan, the nominal $1.1-\mathrm{km}$ value was used for the half height width of the point-spread function. The resulting pixel on each of the three fraction images closest to each AVHRR pixel center was selected by registration to a 40 -by-50 pixel (242000 ha) subimage of NOAA7/AVHRR acquired on July 10, 1984 at about 2:30 PST. The fraction images computed with TM were registered to AVHRR to within about 0.5-pixel (AVHRR) accuracy, using nearest neighbor resampling. The registered fraction data consisted of a quadrilateral of 919 pixels within the 40 -by-50 pixels surrounded by zero fill.

Standard multivariate analysis techniques were employed to fit the mixture model. Using (2), (1) can be rewritten to eliminate one fraction, $F 3$, as

$$
L(p, b)=A 1 * F 1(p)+A 2 * F 2(p)+A 3+c(p, b)
$$

with

$$
\begin{aligned}
& M 1(b)=A 1+A 3 \\
& M 2(b)=A 2+A 3 \\
& M 3(b)=A 3 .
\end{aligned}
$$

Estimates of the mean DN values for each class were thus derived by regressing DN values against the fractions for clearcut, $F 1(p)$, and forest, $F 2(p)$, to compute $A 1, A 2$, and A3 coefficients, and then computing $M 1(b), M 2(b)$, and $M 3(b)$ from (3). In addition, the covariance matrix of the regression residuals, i.e., the values for $e(p, b)$, were estimated using the usual formulas for sample variance (standard deviation squared) for each band and covariances of band pairs.

\section{Unmixing}

The AVHRR pixels were "unmixed" by application of least squares estimation pixel-by-pixel after the signature development. This amounts to least squares fit of (1), where the coefficients to be estimated are now the fractions $F 1, F 2$, and F3, and the "samples" are the image wavebands-the AVHRR DN values associated with the pixel and the class means in each band.

The constraints on estimates for the fractions were included in the unmixing. As with signature development, Condition 2) that the fractions sum to one was algebraically incorporated into the estimation, a least squares fit of

$$
T(p, b)=F 1 * B 1(b)+F 2 * B 2(b)+c(p, b)
$$

with

$$
\begin{aligned}
B 1(b) & =M 1(b)-M 3(b) \\
B 2 & =M 2(b)-M 3(b) \\
T(p, b) & =L(p, b)-M 3(b)
\end{aligned}
$$

to estimate $F 1$ and $F 2$. Then $F 3$ was computed as $1-F 1$ $-F 2$.

This is a generalization of the two-class solution described in [15]. Clearly, the method generalizes to any number $N$ of classes as long as there is enough information in the spectral bands for solution to the system of equations specified by (4). In the case of three classes, this means that there are two bands where the class means are not colinear. A minimum of $N-1$ bands is required that satisfy the condition that the means of each class is not a linear combination, i.e., a weighted vector sum, of other class means [18]. After least squares estimation, nonnegative estimates were ensured by zeroing any negative fractions for a pixel, and then normalizing by the sum of values so that the sum of estimates would still be one. The negative values could arise due to the error term $(c(p, b))$ in (4).

Two least squares techniques were implemented-ordinary least squares (OLS) and generalized least squares (GLS) [19]. Ordinary least squares is a common regression technique and is optimal for cases where the $e(p, b)$ terms are identically and independently distributed. In our application, this condition means that variances of DN values within each of the three forest classes are about the same and are not correlated among bands. The generalized least squares technique is optimized for cases where these conditions do not apply. It was implemented by transforming the AVHRR DN values as described in $|18|$, and then applying OLS. The transformation was computed from the covariance matrix for the $e(p, b)$, so that the transformed data would conform to the OLS conditions. 
TABLE I

AVHRR Signatures of Forfist Classes

\begin{tabular}{|c|c|c|c|c|c|c|c|c|}
\hline \multirow[b]{2}{*}{ BAND } & \multicolumn{3}{|c|}{$1 \quad$ MEANS } & \multicolumn{5}{|c|}{ COVARIANCE } \\
\hline & I CLEARCUT & FOREST & SUCCESSION & 1 & 2 & 3 & 4 & 5 \\
\hline 1 & 174.3 & 55.3 & 65.1 & 13.24 & 13.9 & -23.4 & -6.7 & -5.7 \\
\hline 2 & 1100.9 & 95.3 & 164.0 & 1 & 118.8 & -100.2 & -35.5 & -33.2 \\
\hline 3 & I 331.1 & 642,8 & 614.4 & 1 & & 1004.9 & 437.3 & 362.3 \\
\hline 4 & 210.6 & 319.2 & 313.7 & 1 & & & 240.2 & 194.9 \\
\hline 5 & 246.1 & 336.6 & 331.2 & 1 & & & & 161.3 \\
\hline
\end{tabular}

The unmixing was computationally very simple. While performing a regression analysis of each pixel in an image can, in general, be a daunting task, it is greatly simplified computationally by the fact that the dependent variables (i.e., the class means) were the same for each pixel. The regression part, whether OLS or GLS, of the unmixing therefore was equivalent to a linear transformation of the AVHRR image. Zeroing negative values and renormalizing are also quite simple compared to such alternatives as zeroing negative estimates and computing least squares estimates on fewer variables (in the three class case-one variable), as others have done [15]. This simplified technique was considered adequate because it should give estimates similar to those derived from more sophisticated techniques when negative initial estimates are small in magnitude. When the magnitude of negative initial estimates is large, all estimates will probably be poor as this will only occur when the error term in the model (1) is large or when the model is innapropriate.

\section{RESUlTS}

\section{A. Signature Development}

Table I shows the signatures of the disturbance classes estimated from the Andrews area-919 pixels of AVHRR and class fractions computed with TM classified imagery. These can be interpreted by noting that radiances in the reflectance bands (AVHRR1 and AVHRR2) are approximately increasing linear functions (positive slope) of the digital counts while radiances in the longer wavelength bands (AVHRR3, AVHRR4, and AVHRR5) are approximately decreasing linear functions (negative slope) of the digital counts [16], [20]. Table I, therefore, shows high reflectance in the visible band (band 1) and thermal emittance (bands $3,4,5$ ) of clearcuts compared to vegetated areas. The mature forest is differentiated from regrowth (succession) areas by lower reflectances, especially in the NIR, due to predominance of conifer species in undisturbed areas versus predominance of deciduous plants in disturbed areas. Both vegetation classes are cooler by about $10^{\circ} \mathrm{C}$, estimated by conversion of digital counts to radiance values followed by inversion of Planck's equation [20], than the clearcut areas. These differences among classes are clearly significant, given that they are at least several times the standard deviations for each band (square root of the diagonal elements of the covariance matrix).

Fig. 3 illustrates the similarities between the TM and AVHRR signatures in the reflective bands. The $50 \%$ concentration ellipses for each TM class were constructed from spectral statistics for the 30 sites used to train the classification.

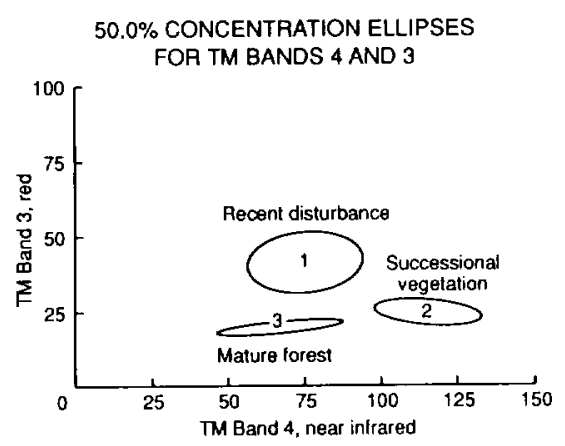

(a)

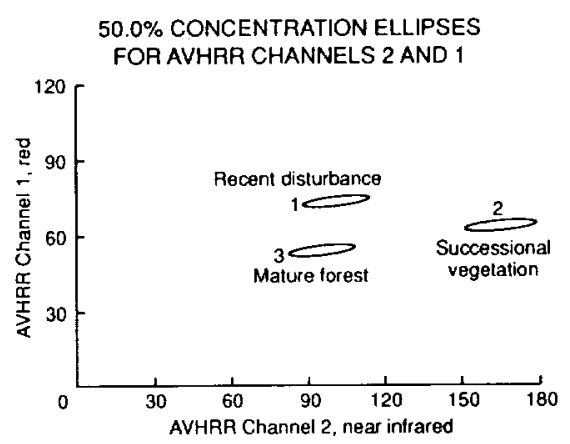

(b)

Fig. 3. $50 \%$ concentration ellipses of the signatures of clearcut, forest, and successional forest in the visual red and near infrared of (a) Landsat TM and (b) AVHRR.

The $50 \%$ concentration ellipses of AVHRR were constructed with the means and covariances of Table I. The ellipses corresponding to the cover classes form triangles; therefore, mixtures of any two disturbance classes are distinct from the third and estimation of mixture fractions appears feasible with only these two bands.

Linear least squares methods are based on the assumption that the distribution of the error term (1) and (4) does not depend on the values of the independent variables [18], [19]. For unmixing, these variables are the mean DN's for each class; therefore, the assumption implies a single covariance matrix for all classes [18]. To test the appropriateness of a single variance-covariance matrix for all classes, in particular equality of variances among classes, the residuals from the signature development regression were analyzed using a technique similar to Levene's test [21] for equality of variance in analysis of variance. First, autocorrelation functions were evaluated to determine an appropriate sampling frequency so that values were uncorrelated, as this is a general assumption for statistical tests of significance. The magnitudes of the autocorrelations were less than their standard error values with a spacing of four or more. The absolute value of the residuals for the pixels in every fifth row and every fifth column were regressed on the class fractions. The regressions of the residuals in AVHRR2 were highly significant $(p=0.03)$ for both clearcut and forest, and somewhat less significant $(p=0.11)$ for the successional class. The regressions for the other bands were somewhat less significant for AVHRR $1(\psi$ values of 0.13 to 0.33 ) and much less significant in the thermal 
TABLE II

Estimattis rRom UnMixingi AVHRR Bandes 1, 2, 3, 4 (WL.S RI:SUITS) COMPARID) TO TM ESTIMATES

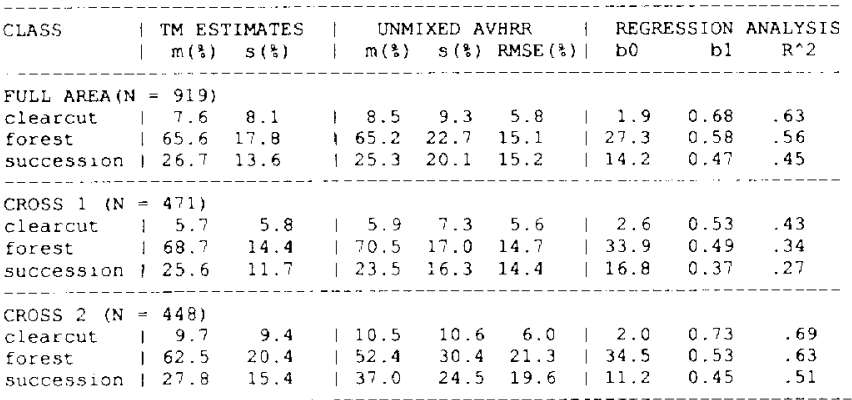

bands. These results suggest that a single covariance matrix adequately represents all three classes for AVHRR 1, 3, 4, 5, but not AVHRR 2. Positive regression coefficients suggest that there is more variation in near-infrared reflectance in clearcuts and regrowth areas than in the undisturbed forest (negative regression coefficient).

\section{B. Unmixing}

The unmixing procedure was tested first by using the signatures reported in Table I to unmix the AVHRR data (bands 1-4). Then the procedure was further tested by splitting the study area roughly into north and south halves and using the north half for signature development and unmixing the south half (CROSS 1) and vice versa (CROSS 2). The results are summarized in Tables II (GLS technique) and III (OLS technique) which show the mean $(m)$ and standard deviation $(s)$ for each class fraction, as estimated by TM and by unmixed AVHRR, the root mean square error (rmse) of the unmixed AVHRR relative to the TM fractions, and the results of regressing the unmixed AVHRR pixels against the convolved TM.

Comparison between the mean TM and AVHRR fractions (Table II) shows that GLS unmixing predicted the composition of the study site fairly well, although there was some confusion between forest and regrowth areas in the southern half of the area. Per-pixel estimates of mixture were not very accurate, however, with rmse values nearly as large as the standard deviation of the TM fractions. This may have been due to effects of registration errors as well as innaccuracies of the unmixed imagery, as the scale of the registration errors $(0.5$ pixel) was similar to the scale of the clearcuts. Still, since the $R$-squared values were highly significant $(p<0.01$ for degrees of freedom approximating the number of pixels divided by 25) the unmixed image generally follows trends in land use, as is also evident in plots of TM and AVHRR fractions along four arbitrarily selected transects through the full test area (Fig. 4).

Comparisons between Tables II and III indicate the generally superior performance of the GLS unmixing procedure relative to OLS. This was expected, given considerable differences between signature variances between bands and correlations between bands indicated in Table I.

The unmixing procedure was further tested with AVHRR bands 1 and 2, as the separability of classes appeared to be
TABLE III

ESTIMATLS FROM UNMIXING AVHRR 1, 2, 3 , 4 (OLS RESUITS) COMPARID TO TM ESTIMATES

\begin{tabular}{|c|c|c|c|c|c|c|c|c|c|c|c|}
\hline CLASS & \multicolumn{3}{|c|}{ TM ESTIMATES } & \multicolumn{4}{|c|}{ UNMIXED AVHRR } & ! & \multicolumn{2}{|r|}{ SS ION } & $\begin{array}{c}\text { ANALYSIS } \\
\mathrm{K}^{\wedge} 2\end{array}$ \\
\hline FULL AREA (? & & 919) & & & & & & & & & \\
\hline clearcut & i & 7.6 & 8. 1 & 1 & 9.7 & 10.5 & 8.4 & 1 & 2.9 & 0.49 & 40 \\
\hline forest & 1 & 65.6 & 17.8 & i & 65.0 & 24.6 & 18.1 & i & 33.7 & 0.49 & .46 \\
\hline succession & I & 26.7 & 13.6 & i & 25.3 & 20.1 & 15.1 & I & 15.4 & 0.45 & .43 \\
\hline CROSS 1 (N & $=$ & 471) & & & & & & & & & \\
\hline clearcut & I & 5.7 & 5.8 & I & 11.8 & 9.1 & 9.9 & 1 & 1.7 & 0.34 & .28 \\
\hline forest & 1 & 68.7 & 14.4 & 1 & 65.3 & 19.5 & 17.9 & I & 44.6 & 0.37 & .25 \\
\hline succession & I & 25.6 & 11.7 & I & 22.8 & 16.0 & 14.4 & 1 & 17.0 & 0.38 & .27 \\
\hline CROSS 2 (N & & 448) & & & & & & & & & \\
\hline clearcut & 1 & 9.7 & 9.4 & I & 7.8 & 10.5 & 7.7 & I & 4.5 & 0.65 & 53 \\
\hline forest. & 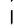 & 62.5 & 20.4 & i & 64.4 & 31.2 & 20.1 & i & 29.8 & 0.51 & .60 \\
\hline succession. & $\mathrm{i}$ & 27.8 & 15.4 & । & 27.8 & 25.1 & 17.7 & । & 15.7 & 0.44 & .51 \\
\hline
\end{tabular}

TABLE IV

ESTIMATES FROM UNMIXING AVHRR 1, 2 COMPARID TO TM ESTIMAIES

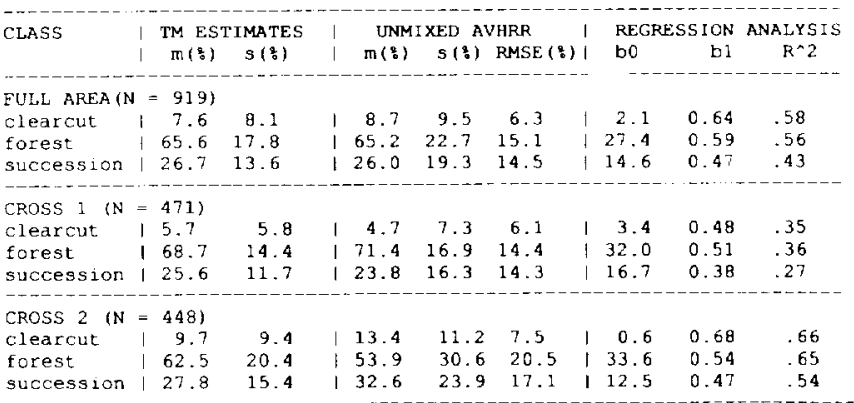

sufficient with just these two reflective bands (Fig. 3). As before, unmixing was tested using Table I signatures on the full study area and using cross validation (CROSS 1 and CROSS 2 ). In this two-band and three class case, the formulas for least squares estimates (either OLS or GLS) are equivalent to the exact solution of (1) and (2), ignoring the error terms. As can be seen by comparing results summarized in Table IV with Tables II and III, the resulting estimates of class fractions were actually better than OLS estimates with AVHRR bands $1-4$ and almost as good as GLS estimates using the four bands. There were small improvements in estimates of the clearcut fractions by including the thermal bands, with increases in $R^{2}$ values for clearcut of $5 \%$ (using Table I signatures), $8 \%$ (CROSS 1 ), and 3\% (CROSS 2) and small degradations in $R^{2}$ of $0 \%$ to $3 \%$ for the forest and regrowth classes.

\section{CONCLUSIONS}

Methods have been developed to extract signatures from AVHRR using finer resolution data for training, and for unmixing AVHRR pixels. These methods are based on constrained least squares and generalized least squares techniques, and are computationally simple and fast.

While thorough testing of procedures at the scale of an AVHRR data set (millions of square kilometers) was beyond the scope of this investigation, the results of assessment with test areas selected from a Landsat scene (hundreds of square kilometers) look promising. The extracted signatures exhibited the expected spectral properties of the classes. Estimates of percentages of the land cover types of the test areas from the unmixed AVHRR were within $10 \%$ of the estimates based on 

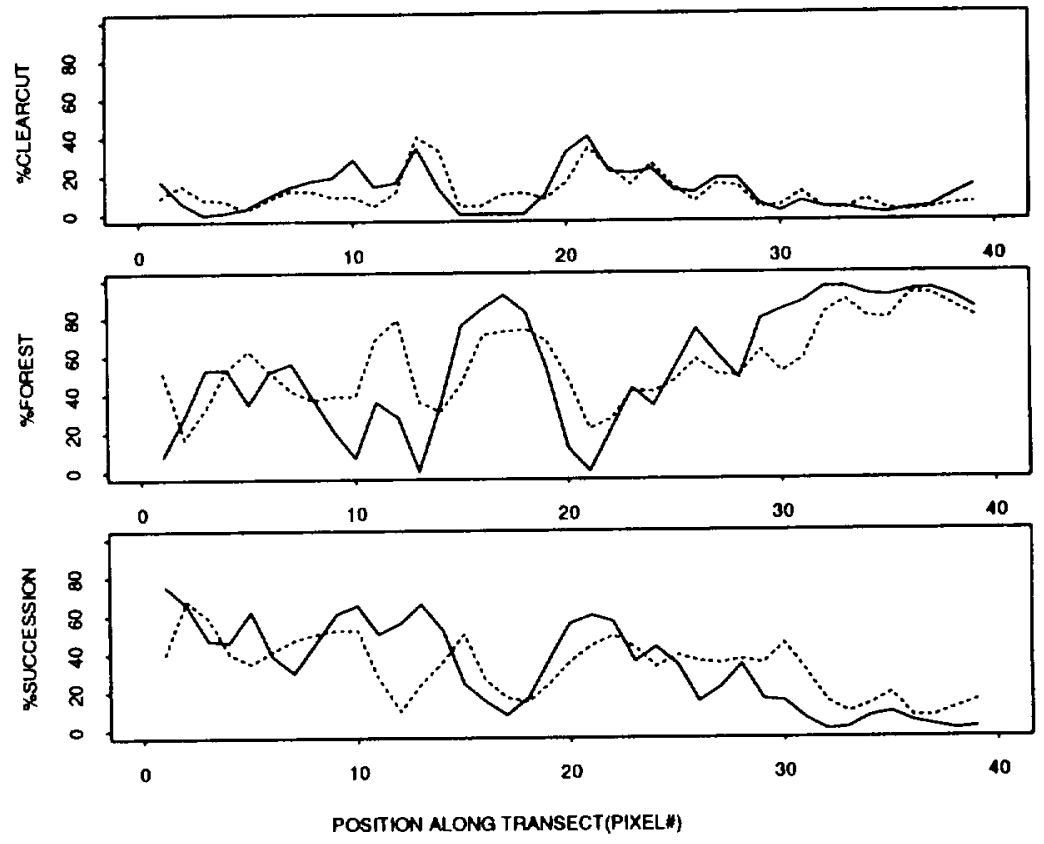

(a)
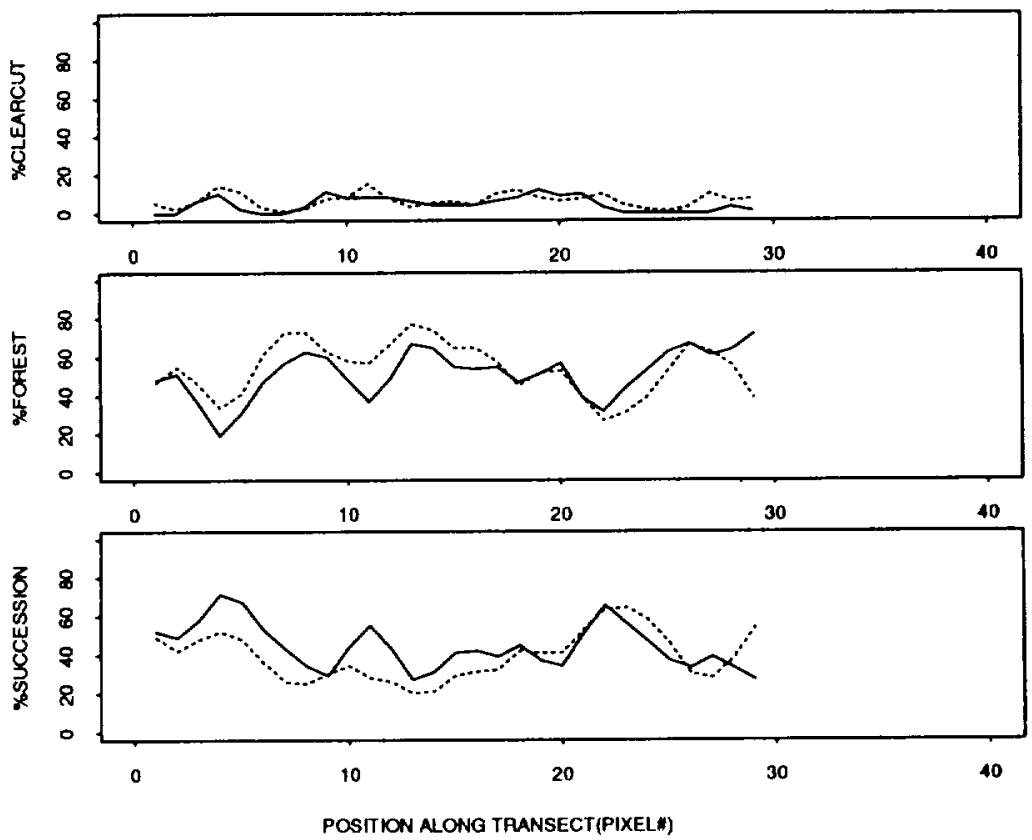

(b)

Fig. 4. Percent cleareut, forest, and successional forest (brush) for pixels along four transects in the imagery as computed with TM (dotted line) and AVHRR (GLS unmixing technique, solid line). (a) 15 th row. (b) 30 th row.

analysis of Landsat TM imagery. The apparent accuracy of estimated percentages at finer (per-pixel) scale were poor.

Including thermal bands in unmixing only minimally improved results when using GLS techniques, and actually degraded the results obtained with only the reflective bands when using OLS techniques. This suggests that thermal information does not contribute much to analysis of land cover. The improvement in results through using GLS rather than OLS with more than the minimal number of bands (number of classes less one, when the solution is exact) suggests
GLS techniques may improve the results of unmixing in applications with other sensors, particularly when the spectra characteristics of the mixing components are variable, as is the case with land cover types.

Application of unmixing to large areas, such as the millions of square kilometers covered in an AVHRR frame, would involve some extra steps in processing. For example, one could consider extending this analysis of forest and clearcuts to a large region in the Pacific NW. This would require masking out nonforest areas, such as valleys and large areas of lava 

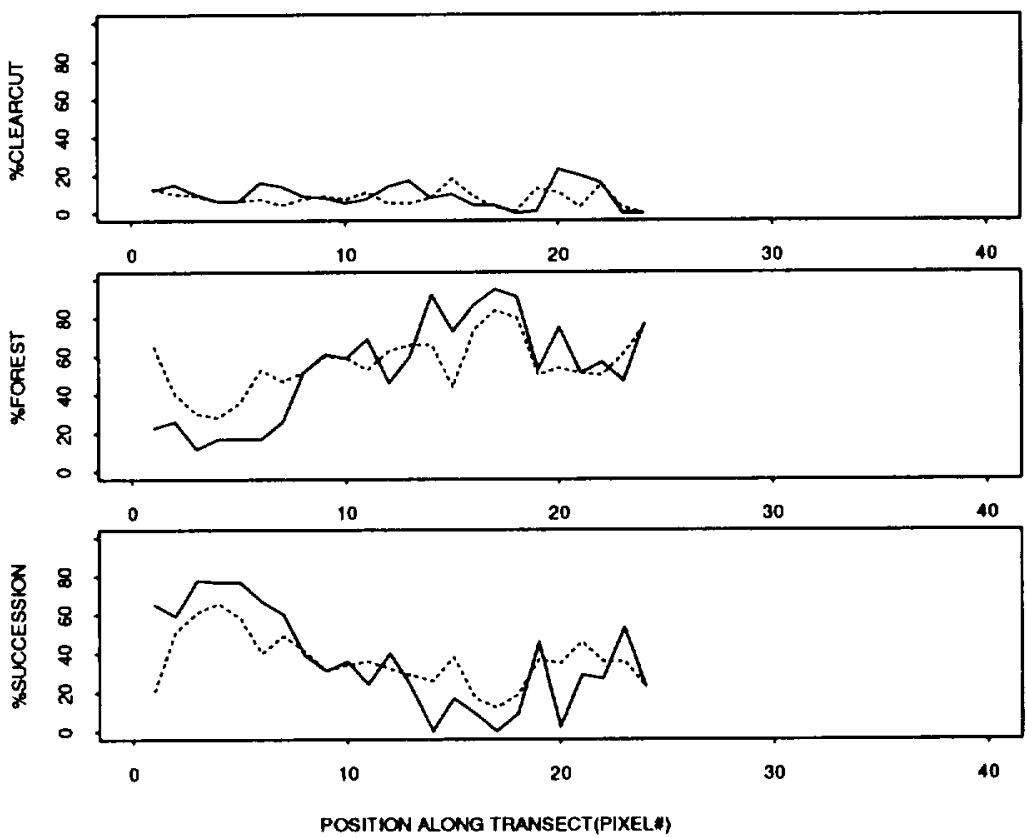

(c)
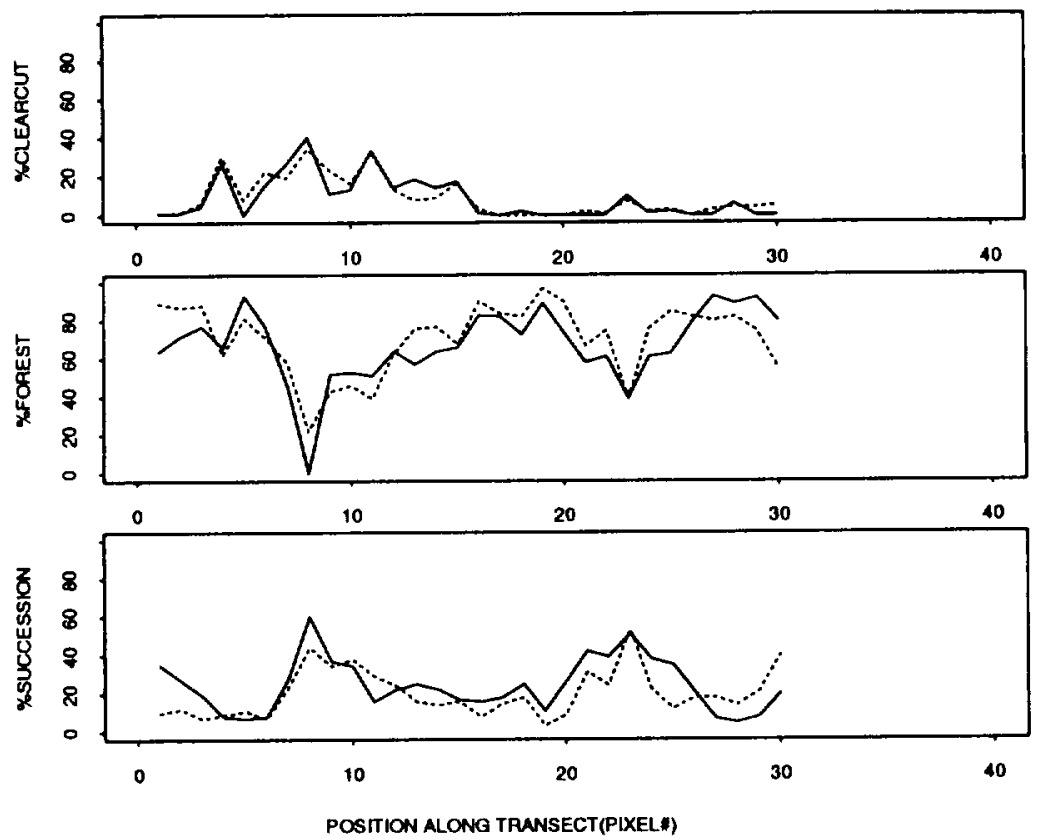

(d)

Fig. 4. Percent clearcut, forest, and successional forest (brush) for pixels along four transects in the imagery as computed with TM (dotted line) and AVHRR (GLS unmixing technique, solid line). (a) Fifteenth column. (b) Thirtieth column.

flows, in order to restrict application of unmixing to areas which can be modeled as mixtures of the three forest classes. Preprocessing the data, for example by applying radiometeric corrections for slope aspect effects, would help to minimize variation in the spectral characteristics of the forest classes across the region. Use of several finer scale images would be advisable for developing representative signatures.

\section{ACKNOWLEDGMENT}

The authors wish to thank L. Pierce of the School of Forestry, University of Montana, for his assistance with collec- tion of field data and L. Satmayo of the U.S. Forest Service, H. J. Andrews Experimental Forest, for providing stand maps and stand history data. We also thank several anonymous referees for their suggestions, which guided the revision of the paper.

\section{REFERENCES}

111 J. R. G. Townshend, G. E. Goff, and C. J. Tucker, "Multitemporal dimensionality of images of normalized difference vegetation index at continental scales," IEEE Trans. Geosci. Remote Sensing, vol. GRS-23, no. 6 , pp. 888-895, 1985.

[2] J. P. Malingreau, "Global vegetation dynamics: satellite observations over Asia," Int. J. Remote' Sensing, vol. 7, no. 9, pp. 1121-1146. 1986. 
[3] R. Nelson, N. Horning, and T. A. Stone, "Determining the rate of forest conversion in Mato Grosso, Brazil, using Landsat MSS and AVHRR data," Int. J. Remote Sensing, vol. 8. no. 12. pp. 1767-1784, 1987.

14] L. R. Iverson, E. A. Cook, and R. L. Graham, "A technique for extrapolating and validating forest cover across large regions-calibrating AVHRR data with TM data," Int. J. Remote Sensing, vol. 10, no. 11, pp. $1805-1812,1989$

15] P. M. Vitousek and J. M. Mellilo, "Nitrate losses from disturbed forests: Patterns and mechanisms," Forest Sci., vol. 25, pp. 605-619, 1979

[6] P. A. Matson and P. M. Vitousek, "Nitrogen mineralization and nitrification potentials following clearcutting in the Hoosier National Forest, Indiana," Forest Sci., vol. 27, pp. 781-791, 1981.

17] P. M. Vitousek and P. A. Matson, "Disturbance, nitrogen availability and nitrogen losses: An experimental study in an intensively managed Loblolly pine plantation," Ecology, vol. 66, pp. 1360-1376, 1985.

181 R. Waring and J. Franklin, "Evergreen coniferous forests, of the Pacific Northwest," Science, vol. 204, pp. 1380-1386.

[9] J. F. Franklin and C. T. Dryness, "A checklist of vascular plants on the H. J. Andrews Experimental Forest, Western Oregon," USDA Forest Res. Note PNW-138, USDA Forest Service, Portland, OR, 1971.

[10] G. Hawk, J. Franklin, W. McKee, and R. Brown, "H. J. Andrews Experimental Forest Stand System: Establishment and use history," Bull. 12. Coniferous Forest Biome U.S./International Biological Program, 79 pp., 1978.

(11) M. A. Spanner, C. A. Hlavka, and L. L. Pierce, "Analysis of forest disturbance using TM and AVHRR data," in Proc. Int Geosci. Remote Sensing, I2th Canadion Symp. Remote Sensing. Vancouver, BC. Canada, 1989, pp. 1387-1390.

[12] H. M. Horwitz, R. F. Nalepka, P. D. Hyde, and J. P. Morgenstern "Estimating the proportions of objects within a single resolution element of a multispectral scanner," in Proc. 7th Int. Symp. Remote Sensing Environ., Ann Arbor, M1, 1971, pp. 1307-1320.

[13] S. G. Ungar and E. Bryant. "Fanning: A classification algorithm for mixture landscapes applied to Landsat data of Maine forests," presented at the 15th Iml. Symp. Remote Sensing Environ.. Ann Arbor, MI, 1981.

$114 \mid$ J. B. Adams, M. O. Smith, and P. E. Johnson, "Spectral mixture modeling: A new analysis of rock and soil types at the Viking Lander l site,"J. Geophis. Res., vol. 91, no, B8, pp. 8098-8112, 1986.

[15] Y. E. Shimahukuro and J. A. Smith, "The least-squares mixing models to generate fraction images derived from remote sensing multispectral data" IEEE Trans. Geosci. Remote Sensing. vol. 29, pp. 16-20, 1991.

[16] J. C. Price, "Calibration of satellite radiometers and the comparison of vegetation indices," Remore Sensing Environ., vol. 21, pp. 15-27, 1987.

[17| C. A. Hlavka, "Simulation of Landsat multispectral scanner spatial resolution with airborne scanner data," NASA Tech. Memo. 86832, 11 pp., 1986.

[18] H. M. Horwitz, J. T. Lewis, and A. P. Pentland, "Estimating proportions of objects from Multispectral Scanner data," Final Rep. NASA CR 141862, ERIM 10960()-13-F. Environ. Res. Inst. Michigan. Ann Arbor. 117 pp., 1975.

1191 A. T. Thisted, "GLM: The general linear model," in Elements of Statistical Computing. New York: Chapman and Hall, 1988, ch. 3

[20] K. B. Kidwell, NOAA Polar Orbiter Data Users Guide. National Oceanic and Atmospheric Administration-National Environmental Satellite, Data and Information Service-National Climatic Data Center-Satellite Data Services Division, Washington DC. July 1991.

1211 H. Levene, "Robust tests for equality of variance," in I. Olkin, Ed.. Contribations to Probability and Statistics. Palo Alto. CA: Stanford Univ. Press. 1960). pp. $278-292$.

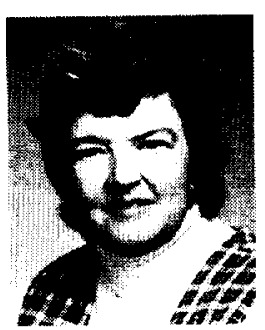

Christine A. Hlavka received the B.S. degree in physics from Pomona College. Pomona, CA. in 1970 and the M.S. degree in statistics from the University of Wisconsin, Madison, in 1976.

She has been a Research Scientist al NASA's Ames Research Center. Moffett Field. CA, since 1980, and has worked on a variety of projects involving remote sensing and signal processing. Her current interests are in image processing techniques for regional ecosystem studies, and the analysis of spatial pattern in imagery of the earth's surface.

Ms. Hlavka is a member of the American Statistical Association.

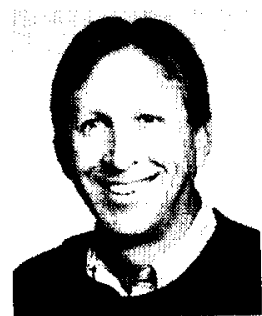

Michael A. Spanner received the B.A. degree in geography from Sonoma Stale Lniversily. sonoma. CA, and the M.A. degree in geography from the University of California, Santa Barhara, specializing in remote sensing.

$\mathrm{He}$ is currently a Senior Research Scientist for TGS Technology, Inc.. in the Earth Systems Science Division at NASA Ames Research Center, Moffelt Field. CA. His current research includes the use of remotely sensed data for the analysis of forest structural and functional characteristics. He has been involved with the FIFE, and HAPEX-II/Sahel experiments, making measurements of atmospheric optical propertics for atmospheric correction of remotely sensed data. He is currently the project manager for a project using remotely sensed data to determine habitats that are susceptible 10 veclor-horne diseases 